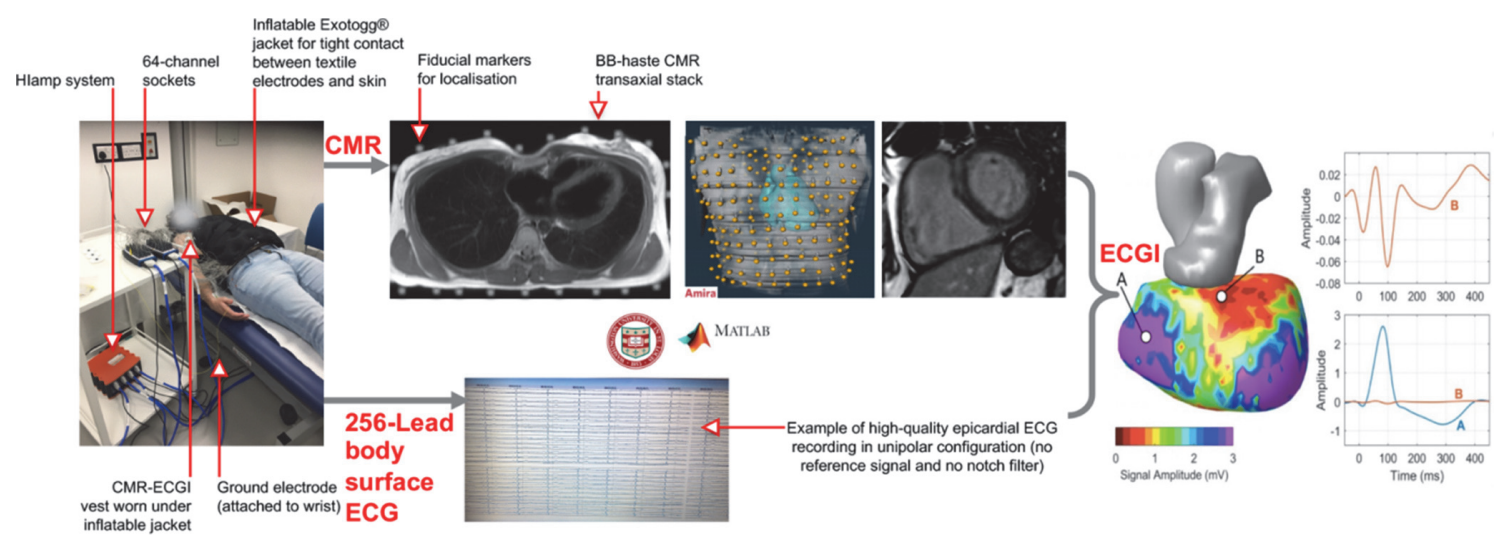

Abstract 12 Figure 1 Agile CMR-ECGI workflow. ECGI recording precedes CMR using Hlamp multi-channel amplifier connected to g.Recorder software at a sampling frequency of $2048 \mathrm{~Hz}$, for 5 mins at rest in the supine position. CMR-ECGI vest montage and doffing is quick with total preparation time per patient $<5$ mins, thanks to the vest design and dry electrode solution. CMR protocol includes transaxial black blood (BB) anatomical stack for electro-anatomical mapping using fiducial markers for co-registration. Electrode-skin surface contact is enhanced using an inflatable jacket. An exemplar activation time isochrone map is shown for a 75-year study member with midwall late gadolinium enhancement in the mid anteroseptal: note the matching zone of fractionation and low-amplitude in the unipolar electrograms. CMR=cardiovascular magnetic resonance; $\mathrm{ECGI}=$ electrocardiographic imaging.

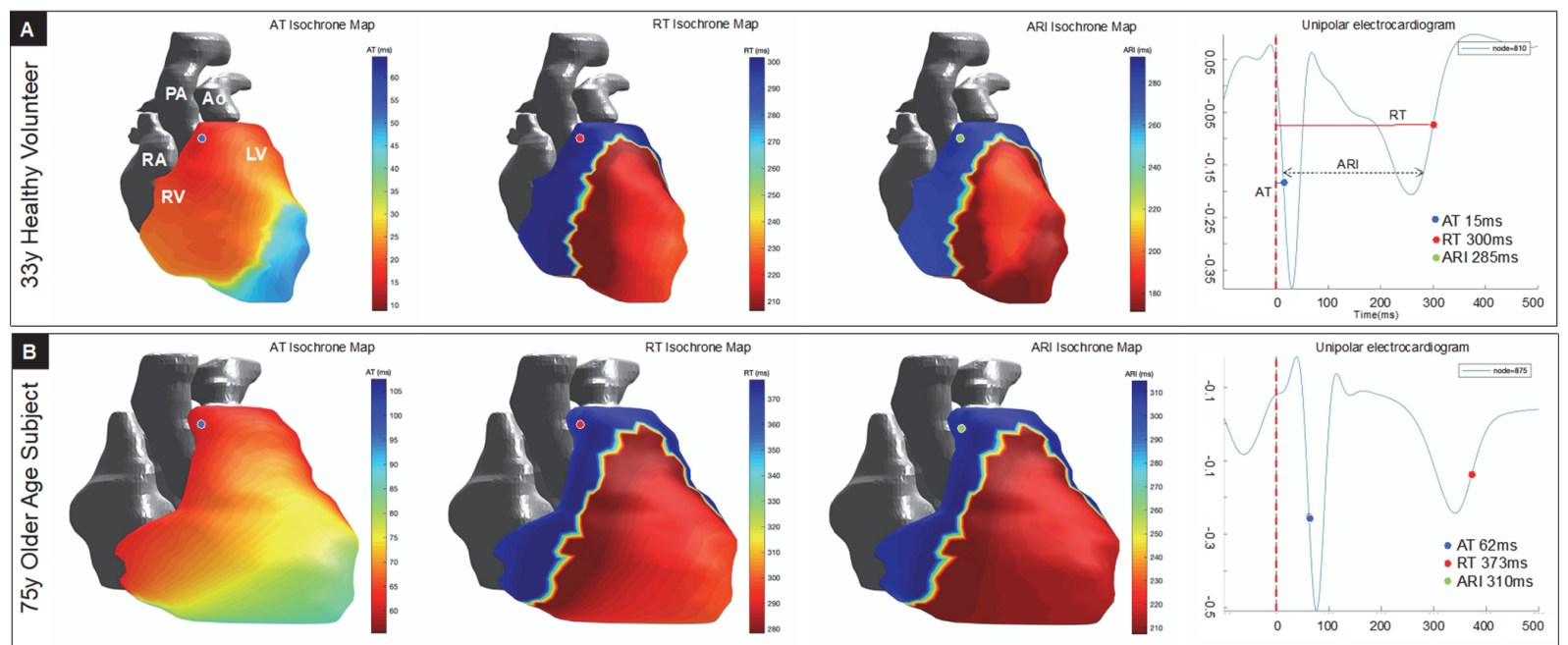

Abstract 12 Figure 2 Activation and recovery time isochrone maps arising from CMR-ECGI in a healthy 33-year old female volunteer (Top panel A) and in a 75-year-old male with no significant past medical history (Bottom panel B). Unipolar electrocardiograms are shown for each participant representing the point of initial activation. Of note is the prolonged activation time, repolarisation time and activation recovery interval of the older participant when compared to the young control. ARI=activation recovery interval; AT=activation time; RT=repolarisation time; $y=y e a r s$.

prolongation (respectively $\beta=0.3 \mathrm{~ms}[95 \%$ confidence interval $0.01-0.64] p=0.048 ; \beta=6.0 \mathrm{~ms}[1.85-10.05] p=0.006$; $\beta=4.9$ ms $[0.22-9.60] p=0.041)$ and, $\mathrm{T}_{2}$ and ECV with ARI prolongation $(\beta=5.9 \mathrm{~ms}[1.88-9.92] p=0.005 ; \beta=4.8$ ms [0.40-0.12] $p=0.033)$. All associations persisted after adjusting for LVEF.

Conclusion The normal electrophysiological sequence of activation and repolarisation in the human heart changes markedly with ageing and may be explained by CMR-detected myocardial substrate changes consisting of low-grade inflammation and diffuse fibrosis. Our high-throughput and reusable CMR-ECGI solution has the ability to provide unprecedented insights into the pathophysiology of arrhythmogenesis beyond conventional measures of cardiac structure and function.

\section{THE ASSOCIATION BETWEEN CARDIOVASCULAR RISK FACTORS AND LEFT ATRIAL STRUCTURE AND PHASIC FUNCTION}

${ }^{1,2}$ Mihir M Sanghvi, ${ }^{1}$ Jose D Vargas, ${ }^{3}$ Avan Suinesiaputra, ${ }^{3}$ Rodrigo Bonazzola, ${ }^{3}$ Rahman Attar, ${ }^{3}$ Nishant Ravikumar, ${ }^{1}$ Mark Wong, ${ }^{1}$ Hafiz Naderi, ${ }^{1,2}$ Steffen E Petersen, ${ }^{3}$ Alejandro F Frangi, ${ }^{1,2}$ Nay Aung. ${ }^{1}$ Centre for Advanced Cardiovascular Imaging, William Harvey Research Institute, Queen Mary University of London, Charterhouse Square, London, EC1M 6BQ; ${ }^{2}$ Barts Heart Centre, Barts Health NHS Trust, West Smithfield, London, EC1A 7BE; ${ }^{3}$ School of Computing, University of Leeds, Woodhouse, Leeds, LS2 9JT

\subsection{6/heartjnl-2021-BSCMR.13}

Introduction Changes in left atrial (LA) phasic function defined as reservoir function (filling), conduit function (passive emptying), booster function (active emptying) (figure 1A) are thought to precede structural changes (dilatation) of the 
LA and therefore may be a sensitive biomarker in heralding cardiovascular disease.

This study is the first to examine the association between traditional cardiovascular risk factors and LA structure and function in a large population.

Methods LA volumes were calculated from four- and twochamber CMR cines performed as part of the UK Biobank imaging substudy using the biplane method. To determine phasic function, the LA was automatically contoured in the fourchamber views in all fifty frames of the cardiac cycle using a three-dimensional sparse active shape model (SPASM) with parameters estimated using a deep neural network. A time-volume curve across the cardiac cycle was plotted with a smooth line of best fit (figure 1B). Quality control was performed manually on an initial subset of cases, following which an automated tool was implemented. Utilising the presence of local maxima and minima, LA maximal volume (LAmax), LA minimal (LAmin) volume and LA pre-atrial contraction (LApre) were determined, allowing derivation of total emptying fraction, conduit function and booster function.

Cardiovascular risk factors considered were: age, sex, ethnicity, height, BMI, hypertension, diabetes, hyperlipidaemia, physical activity (metabolic equivalent [MET] minutes/week) and smoking. Multivariable linear regression models were fitted, adjusting for all risk factors with LA parameters as dependent variables.

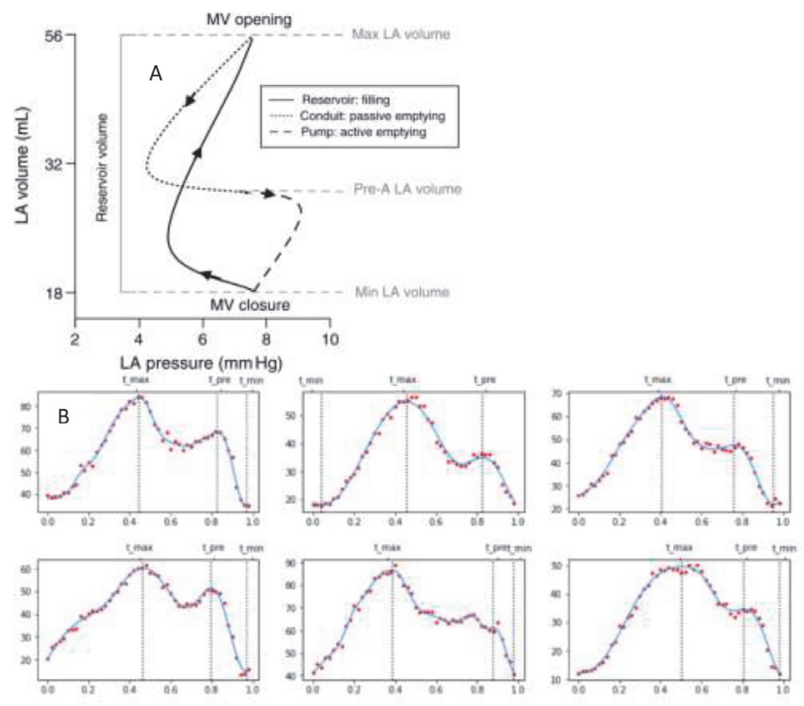

Abstract 13 Figure 1 (A) schematic of atrial phasic function during cardiac cycle. (B) Exemplars of time-volume curves from which atrial function can be derived. $x$-axis represents LA volume in $\mathrm{ml}, \mathrm{y}$-axis is cardiac cycle normalised between 0 and 1 . Functional parameters are derived as follows:

Emptying fraction $=\left(\right.$ LA_max $\left.-L A \_m i n\right) / L A \_m a x$

Conduit function $=\left(\right.$ LA_max $\left.-L A \_p r e\right) / L A \_m a x$

Booster pump $=($ LA_pre - LA_min)/LA_pre

LA size

BMI, HTN, Age = large LA min

BMI, HTN, Age, PA = larger LA max

Diabetes $=$ smaller LA max

Emptying volume and total emptying fraction

BMI, HTN, age = higher emptying volume

$\mathrm{BMI}, \mathrm{HTN}$, age, PA = lower emptying fraction

Conduit function

BMI, HTN, raised chol, smoking $=$ lower conduit function

Booster function

BMI, HTN, age,
Abstract 13 Table 1 Association between cardiovascular risk factors and LA structural and functional parameters

\begin{tabular}{llll}
\hline & Beta & $\begin{array}{l}\text { 95\% confidence } \\
\text { interval }\end{array}$ & p-value \\
\hline LA max (ml) & & & \\
Hypertension & 2.4 & $2.0,2.8$ & $2.3 \times 10^{-33}$ \\
BMI & 1.3 & $1.1,1.4$ & $1.07 \times 10^{-42}$ \\
Diabetes & -1.5 & $-2.2,0.7$ & 0.0001 \\
Total emptying & & & \\
fraction & & & $1.47 \times 10^{-89}$ \\
Hypertension & -0.009 & $-0.01,-0.007$ & $2.77 \times 10^{-18}$ \\
BMI & -0.009 & $-0.01,-0.008$ & $1.15 \times 10^{-136}$ \\
Conduit function & & & $3.85 \times 10^{-05}$ \\
Hypertension & -0.004 & $-0.005,-0.002$ & $9.54 \times 10^{-07}$ \\
BMI & -0.01 & $-0.011,0.009$ & \\
Cigarette smoking & -0.01 & $-0.014,-0.006$ & $1.20 \times 10^{-14}$ \\
Booster pump function & & & $8.89 \times 10^{-31}$ \\
Hypertension & 0.009 & $-0.011,-0.007$ & 0.003 \\
BMI & -0.006 & $-0.007,-0.005$ & \\
Cigarette smoking & -0.003 & $-0.005,-0.001$ & \\
\hline
\end{tabular}

Results After quality control, 44,957 participants were included in the analysis. The mean age was $64 \pm 8,51.8 \%$ of the cohort were female. Regression analysis is detailed in table 1. Hypertension and increasing BMI were significantly associated with lower total emptying fraction, conduit function and booster pump function. Cigarette smoking also resulted in reduced conduit function and booster pump function. Hypertension and BMI were associated with larger LAmax volumes.

Beta coefficients, 95\% confidence intervals and p-values estimating association of risk factors with LA parameters in multivariable linear regression models. Co-variates included in models are as follows: age, sex, ethnicity, height, BMI, hypertension, diabetes, hyperlipidaemia, total MET minutes/week, cigarette smoking.

Conclusion This is the first study to calculate time-volume curves to derive markers of atrial function in a large cohort. We demonstrate that hypertension and increasing BMI - risk factors that are heavily implicated in diastolic dysfunction are most frequently associated with alterations in LA phasic function.

\section{THE PRESENCE OF DIABETES AS A COMORBIDITY ADVERSELY AFFECTS THE PHENOTYPIC EXPRESSION OF HYPERTROPHIC CARDIOMYOPATHY}

${ }^{1}$ Nicholas Jex, ${ }^{1}$ Amrit Chowdhary, ${ }^{1}$ Sharmaine Thirunavukarasu, ${ }^{2}$ Henry Procter, ${ }^{2}$ Anshuman Sengupta, ${ }^{1}$ Pavithra Natarajan, ${ }^{2}$ Ana-Maria Poenar, ${ }^{1}$ Peter Swoboda, ${ }^{3}$ Hui Xue, ${ }^{1}$ Richard M Cubbon, ${ }^{3}$ Peter Kellman, ${ }^{1}$ John P Greenwood, 'Sven Plein, ${ }^{2}$ Stephen Page, ${ }^{1}$ Eylem Levelt. 'University of Leeds, Multidisciplinary Cardiovascular Research Centre and Biomedical Imaging Science Department, Leeds Institute of Cardiovascular and Metabolic Medicine, LS2 9JT, UK; ' 2 Leeds Teaching Hospitals NHS Trust, Department of Cardiology, Leeds, LS1 3EX, UK; ${ }^{3}$ National Heart, Lung, and Blood Institute, National Institutes of Health, DHHS, 10 Center Drive MSC-1061, Bethesda, MD, 20892, USA

\subsection{6/heartjnl-2021-BSCMR.14}

Background Type 2 diabetes mellitus (DM) is associated with worsened clinical outcomes in hypertrophic cardiomyopathy (HCM) patients, although the mechanisms of this association are incompletely understood. 